\title{
Aspectos reproductivos de Grapsus grapsus (Decapoda: Grapsidae) en islas del sureste del Golfo de California
}

\author{
Yecenia Gutiérrez-Rubio ${ }^{凶(\mathbb{0})}$ M.Sc; Juan F. Arzola-González ${ }^{*}{ }^{凶(0)}$ Ph.D; Raúl Pérez-González ${ }^{1} \bowtie(\mathbb{0})$ Ph.D; \\ Guillermo Rodríguez-Domínguez ${ }^{\square}$ (1) Ph.D; José Salgado-Barragán ${ }^{2} \bowtie(\mathbb{0}$ Ph.D; \\ Jorge S. Ramírez-Pérez ${ }^{\square(\mathbb{E}}$ Ph.D; Adrián González-Castillo ${ }^{\circledR}$ Ph.D.
}

\begin{abstract}
${ }^{1}$ Universidad Autónoma de Sinaloa. Facultad de Ciencias del Mar. Doctorado en Ciencias en Recursos Acuáticos. Mazatlán, Sinaloa, México.

2Universidad Nacional Autónoma de México. Instituto de Ciencias del Mar y Limnología. Joel Montes Camarena, Cerro del Vigía. Mazatlán, Sinaloa, México.

3Universidad Politécnica de Sinaloa. Carretera Municipal Libre Mazatlán Las Higueras, Mazatlán, Sinaloa, México.

Correspondencia: farzola@uas.edu.mx
\end{abstract}

Recibido: Marzo 2020; Aceptado: Agosto 2020; Publicado: Noviembre 2020.

\section{RESUMEN}

Objetivo. Se analizó la proporción de sexos, hembras ovígeras, talla de primera madurez sexual y fecundidad del cangrejo roca Grapsus grapsus en islas Lobos, Venados y Pájaros (sureste del Golfo de California). Material y métodos. Los muestreos fueron mensuales entre marzo 2011 y febrero 2012, las colectas fueron nocturnas durante la bajamar, se obtuvieron en un cuadrante $\left(25 \mathrm{~m}^{2}\right)$ por isla 30 organismos al azar, se les determinó el AN $(\mathrm{mm})$ y PT $(\mathrm{g})$. Se estimó la proporción de sexos y talla de primera madurez sexual $\left(\mathrm{AN}_{50 \%}\right)$, se analizaron en hembras grávidas, las fases embrionarias y la fecundidad (método gravimétrico). Resultados. La proporción de M:H fue 1:1.3. La talla media de primera madurez fue $\mathrm{AN}_{50 \%} 34.9 \mathrm{~mm}$. Es evidente la presencia de hembras ovígeras (71.3\%) y todas las fases embrionarias, la fase rojo-naranja fue la mayor representada en $48 \%$. La variación del diámetro del huevo fue 1.1 a $5 \mu \mathrm{m}$ y el promedio de $3.05 \mu \mathrm{m}$. La fecundidad media fue 24339.3 cigotos. El máximo y mínimo peso de hembras ovígeras fue 69.9 y $15.2 \mathrm{~g}$, respectivamente. Conclusiones. Con base a las características biológicas del recurso tales como la proporción de sexos, hembras ovígeras, talla de primera madurez sexual y fecundidad en la población de G. grapsus, representa un efecto favorable en su posterior administración y manejo de este recurso en esta zona.

Palabras clave: Aspectos reproductivos; Grapsus grapsus; islas; Pacífico mexicano (Fuente: CAB Thesaurus, USDA).

\section{ABSTRACT}

Objetive. The sex ratio, ovigerous females, first sexual maturity lengths and fecundity of Grapsus grapsus crab in the Lobos, Venados and Pajaros islands (southeastern Gulf of California) were analyzed. Materials and methods. Sampling was monthly between March 2011 and February 2012, collections were nocturnal at low tide, 30 crabs were collected in a quadrant $\left(25 \mathrm{~m}^{2}\right)$ for island and

Como citar (Vancouver).

Gutiérrez-Rubio Y, Arzola-González JF, Pérez-González R, Rodríguez-Domínguez G, Salgado-Barragán J, Ramírez-Pérez JS, González-Castillo A. Aspectos reproductivos de Grapsus grapsus (Decapoda: Grapsidae) en islas del sureste del Golfo de California . Rev MVZ Córdoba. 2021; 26(1):e1953. https://doi.org/10.21897/rmvz.1953 
sampling. AN ( $\mathrm{mm}$ ) and PT $(\mathrm{g})$ were determined. The proportion of sexes and size of first sexual maturity $\left(\mathrm{AN}_{50 \%}\right)$ was estimated, in ovigerous females, embryonic stages and fecundity (gravimetric method). Results. The sex ratio of $\mathrm{M}: \mathrm{H}$ was $1: 1.3$. The average maturity was $\mathrm{AN}_{50 \%} 34.9 \mathrm{~mm}$. It is evident the presence of ovigerous females (71.3\%) and all the embryonic phases, $48 \%$ of ovigerous was red-orange phase. Eggs diameter from 1.1 to $5 \mu \mathrm{m}$, with an averange diameter of $2.05 \mu \mathrm{m}$. The mean fecundity was 24339.3 eggs. The maximum and minimum weight per female egg was 69.9 and $15.2 \mathrm{~g}$. Conclusions. Based on the biological characteristics of the sex ratio, ovigerous females, first sexual maturity lengths and fecundity of G. grapsus, represent a favorable effect on its subsequent administration and management of this resource in this area.

Keywords: Reproductive aspects; Grapsus grapsus; islands; Mexican Pacific (Source: CAB Thesaurus, USDA).

\section{INTRODUCCIÓN}

Los crustáceos tienen gran importancia para el hombre desde el punto de vista alimenticio y económico. Comprenden un recurso que aporta una cantidad considerable de divisas para México por concepto de exportaciones. Además, muchas especies de crustáceos son consideradas como reguladores ecológicos, por su papel como consumidores o porque forman parte de la dieta de otras especies, muchas de importancia comercial (1). Los crustáceos son habitantes comunes de los ecosistemas marinos y costeros, además son un grupo abundante y muy diverso de las comunidades intermareales de las islas $(2,3)$.

Cuando los pescadores ribereños de la costa de Sinaloa disminuyen la captura de los recursos que explotan regularmente o que no pueden ser explotados durante los periodos de veda, se dedican a otras actividades, como la agricultura (4), o a la extracción de recursos alternativos. Entre ellos, por su accesibilidad y fácil captura, destacan algunas especies de crustáceos que se pueden recolectar en aguas someras o en la franja intermareal. Una de estas especies es el cangrejo roca Grapsus grapsus (Linnaeus, 1758), que es usado localmente como complemento para la alimentación familiar (5) y como carnada para la pesca de escama durante todo el año (2).

El cangrejo roca, G. grapsus es una especie poco estudiada, a pesar de su amplia distribución. Se localiza en playas rocosas del Atlántico desde Florida hasta Brasil, y del Pacífico americano, desde California hasta Chile, incluyendo las islas Galápagos $(6,7)$ e islas del Estado de Sinaloa (2). Los aspectos reproductivos del cangrejo roca fueron analizados en islas (3) y bahía (9) del Atlántico.
En el noroeste de México, los estudios que incluyen a $G$. grapsus solamente se refieren a la especie como parte de colecciones de referencia, aportando datos taxonómicos y del hábitat (2). En las islas del Estado de Sinaloa, las investigaciones sobre aspectos reproductivos en G. grapsus son limitadas, situación muy distinta para otros crustáceos decápodos donde se han analizado diversos aspectos biológicos como langosta espinosa Panulirus gracilis (9) y jaibas Callinectes bellicossus (10) y Callinectes arcuatus (11) en el Pacífico mexicano.

Este trabajo se realizó con la finalidad de obtener información sobre la proporción de sexos, talla de primera madurez y fecundidad de G. grapsus en la zona intermareal de las islas Lobos, Venados y Pájaros del sureste del Golfo de California, México.

\section{MATERIAL Y MÉTODOS}

Estaciones de muestreo y recolectas: Las islas Lobos, Venados y Pájaros, se localizan frente a bahía de Mazatlán, Sinaloa, México entre

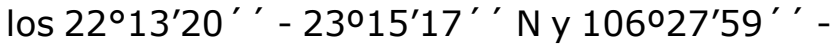
$106^{\circ} 28^{\prime} 41^{\prime}$ ' ' W, al sureste del Golfo de California (Figura 1). Se realizaron muestreos mensuales entre marzo 2012 y febrero 2013 en estaciones georeferenciadas con GPS de la zona intermareal en las islas mencionadas.

La isla Lobos presenta la mayor cantidad y tamaño de rocas en la franja litoral (12). La isla Venados presenta la zona de playa más alargada y son menores los promontorios rocosos y arcillosos, sin embargo, cuando se presenta la bajamar, quedan al descubierto una gran cantidad de rocas. La isla Pájaros, resalta por una amplia zona rocosa-arenosa (4). 


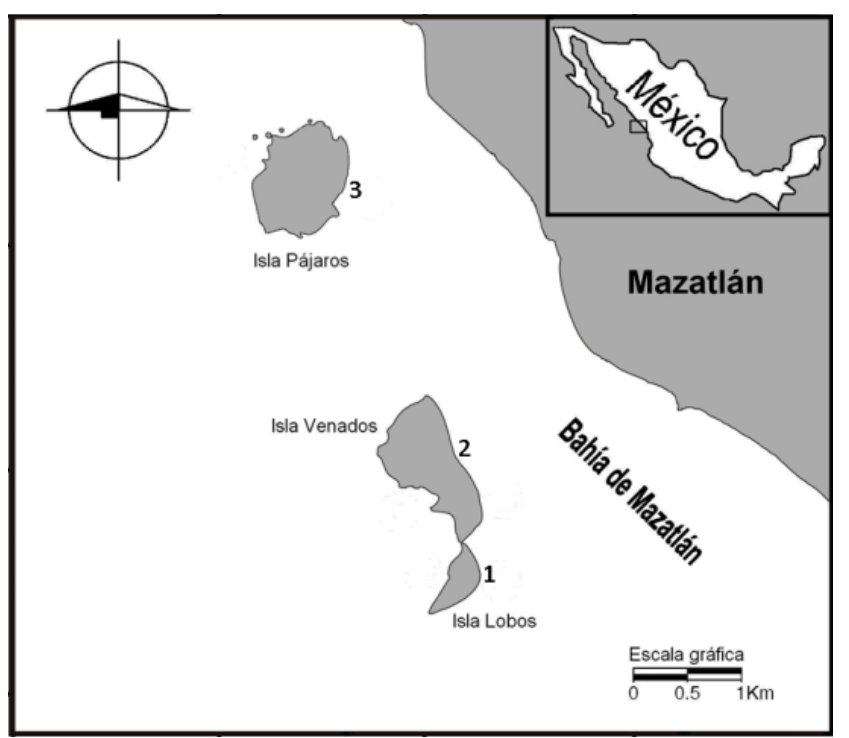

Figura 1. Localización del área de estudio.

Los muestreos de cangrejos fueron nocturnos durante las mareas de sicigia en la fase de bajamar, la colecta de organismos se realizó dentro de un transecto de $2 \times 25 \mathrm{~m}$ paralelo a la línea de costa y con el límite inferior en la línea de bajamar de cada estación (3); 30 organismos de G. grapsus por isla fueron extraídos de forma directa y completamente al azar (procurando extraer los organismos sin selectividad de tallas ni sexos), estos se depositaron en bolsas de plástico con agua de mar y se transportaron al laboratorio en una hielera. A los organismos se les determinó el ancho total (AN) con un vernier digital $(0.01 \mathrm{~mm})$ y su peso total $(\mathrm{PT})$ con una balanza digital (0.1 $\mathrm{g}$ de sensibilidad).

\section{Diferenciación sexual, proporción de sexos} y talla de primera madurez sexual: Los cangrejos braquiuros sexos se diferencian por dimorfismo sexual, los machos presentan un abdomen estrecho, y las hembras adultas muy ancho, este abdomen cubre la mayor parte del esternón de hembras adultas, además, en hembras juveniles y maduras el sexo se determina por la presencia de pleópodos birrámeos, mientras en machos solamente por un par de pleópodos unirrámeos. Se estimó la proporción de sexos y se verificó la proporción de los datos con la prueba de $X^{2}(13)$ y se obtuvieron los porcentajes de hembras ovígeras y no ovígeras. Se determinaron los porcentajes entre las fases de desarrollo embrionario: huevos a) Naranja, b) Rojo-naranja, c) Café claro y d) Café oscuro $(3,8,14)$. La talla de primera madurez $\left(\mathrm{AN}_{50 \%}\right)$ se estimó por el método logístico (11), el cual se basa en el conteo de hembras inmaduras y maduras por cada intervalo de talla.
Fecundidad: Se estimó en hembras grávidas mediante el método gravimétrico (15); la masa ovígera (huevos) fue retirada por cada hembra y fue colocada en pequeñas charolas de papel aluminio previamente pesadas y etiquetadas, las huevas fueron pesadas en fresco en una balanza digital ( $\pm 0.001 \mathrm{~g}$ de sensibilidad). Las masas ovígeras fueron depositadas en cajas de Petri y después fueron secadas en un horno a una temperatura de $50^{\circ} \mathrm{C}$ por 30 minutos, dentro de este tiempo, los huevecillos fueron removidos en las cajas con la finalidad de obtener un mejor secado de estos.

Después del secado, los cigotos fueron depositados en charolas de aluminio y se pesaron nuevamente para determinar el peso seco de la masa ovígera. De cada hueva seca, se obtuvieron al azar tres submuestras de $0.5 \mathrm{~g}$ de cigotos y se colocaron por separado en cajas de Petri. Con un microscopio estereoscópico se contabilizaron los huevos contenidos en cada submuestra y, se obtuvo el promedio del número de cigotos. Finalmente, la media de la cantidad de huevos en $0.5 \mathrm{~g}$ fue extrapolada al peso total de cada hueva seca, obteniendo así, el número total de huevos (fecundidad) por cada hembra ovígera (15).

\section{RESULTADOS}

En total, se analizaron 926 cangrejos roca de los cuales, 407 fueron hembras (44\%) y 519 machos (56\%). Se presentaron diferencias significativas en tallas y pesos entre las islas (Kruskal-Wallis, $\mathrm{p}<0.015$ ) (Tabla 1). La proporción de sexos $\mathrm{M}: \mathrm{H}$ fue $1: 1.13\left(X^{2}=15.2, \mathrm{p}<0.05\right)$.

Tabla 1. Prueba de comparaciones múltiples de Dunn para AN (mm) de G. grapsus en la zona intermareal de las islas del sureste del Golfo de California.

\begin{tabular}{cccc}
\hline \multicolumn{4}{c}{ Isla } \\
\hline Lobos & Venados & Pájaros \\
\hline AN (mm) & $39.91_{\mathrm{b}}$ & $41.47_{\mathrm{ab}}$ & $42.80_{\mathrm{a}}$ \\
PT (g) & $28.42_{\mathrm{b}}$ & $32.29_{\mathrm{ab}}$ & $33.75_{\mathrm{a}}$ \\
\hline
\end{tabular}

Es evidente que la variación entre los sexos, está relacionado con la fase reproductiva de las hembras, la alta incidencia de hembras en etapa ovígeras $(71.3 \%)$ durante todo el estudio, es indicativa de que las hembras de G. grapsus se reproducen constantemente en esta zona (Figura 2 ). Además, en el análisis de los huevecillos se 
observaron todas las fases embrionarias a lo largo del estudio. La fase de desarrollo de cigotos naranja-rojo, registró el mayor porcentaje con $48.8 \%$, y el menor porcentaje correspondió a hembras con hueva color café-oscuro con $26.4 \%$ (Figura 3). La variación del diámetro de los huevos fue entre 1.1 y $5 \mu \mathrm{m}$ con un promedio de $3.05 \mu \mathrm{m}$.

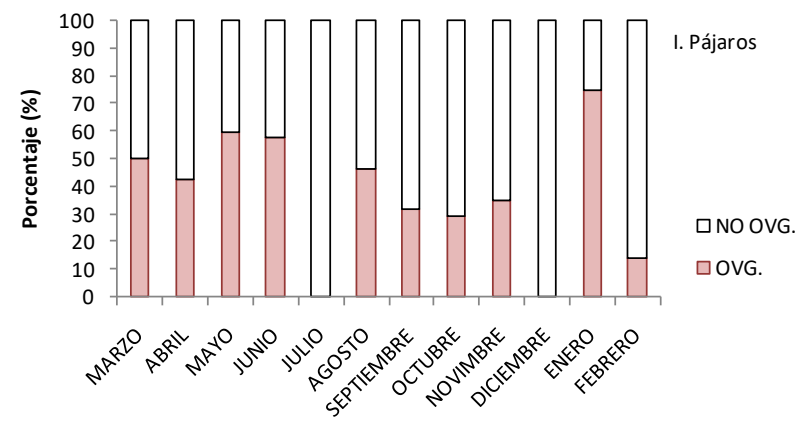

Tiempo (meses)

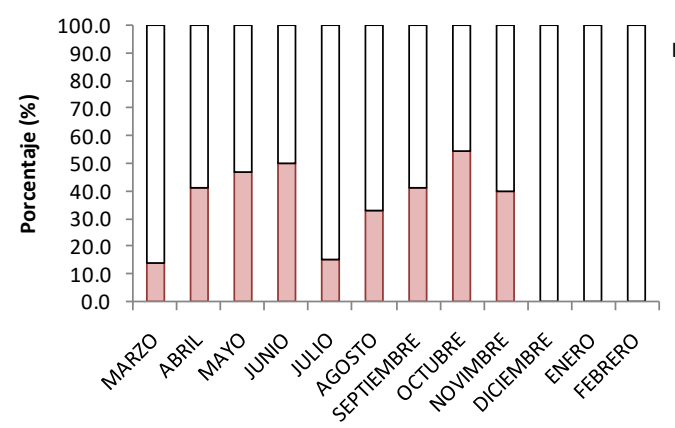

Tiempo (meses)

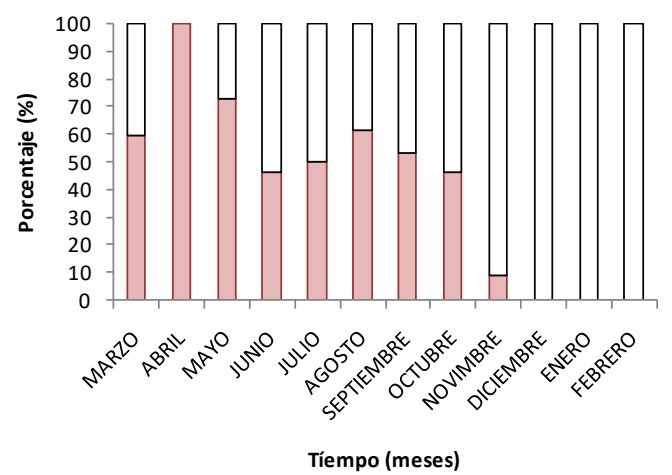

I. Venados

$\square$ NO OVG. $\square$ OVG.

Figura 2. Variación mensual de la proporción de hembras ovígeras y no ovígeras de G. grapsus en la zona intermareal de las islas del sureste del Golfo de California.

La talla de primera madurez de hembras correspondió a $34.87 \mathrm{~mm}$ de AN (Figura 4). La fecundidad media de 24,339.3 huevos, con un máximo de $42,827.5$ (isla Venados) y mínimo de $12,682.4$ huevos (islas Venados y Pájaros). El peso máximo y mínimo de hembras ovígeras fue de 69.9 y $15.2 \mathrm{~g}$, respectivamente (Tabla 2).

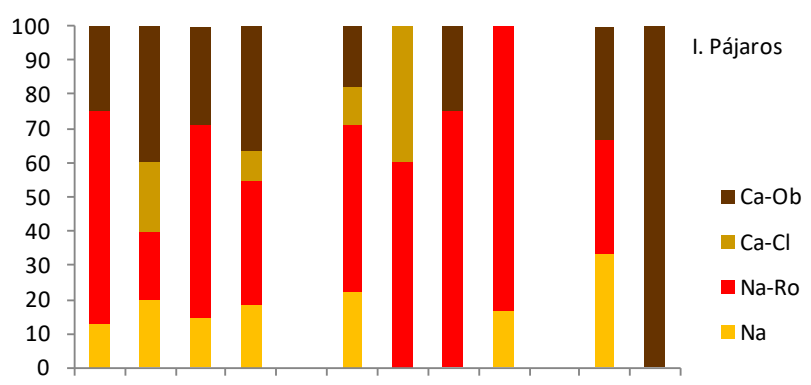

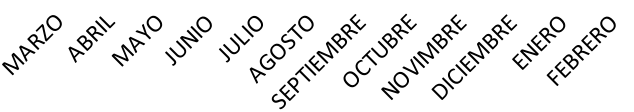
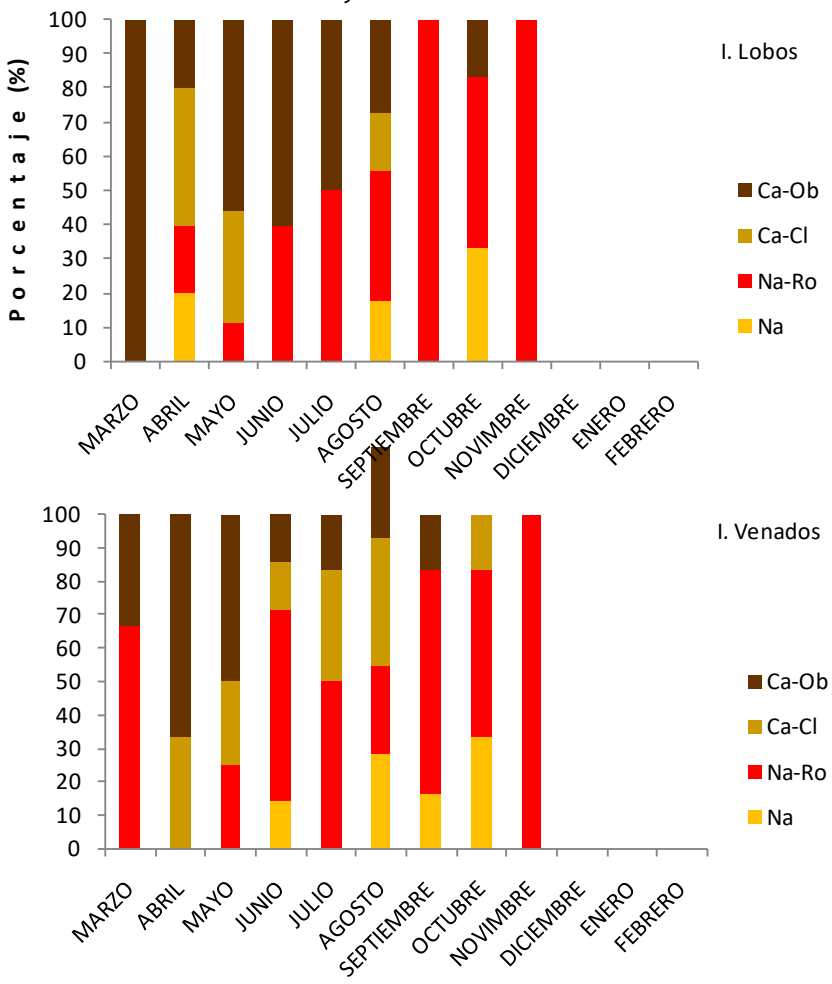

Tiempo (meses)

Figura 3. Frecuencia en porcentaje de fases sexuales en hembras de G. grapsus en la zona intermareal de las islas del sureste del Golfo de California ( $\mathrm{Ca}-\mathrm{Ob}=$ Huevos café obscuro, $\mathrm{Ca}-\mathrm{Cl}=$ Huevos café Claro, Na-Ro=Huevos naranja roja y $\mathrm{Na}=$ Huevos Naranja).

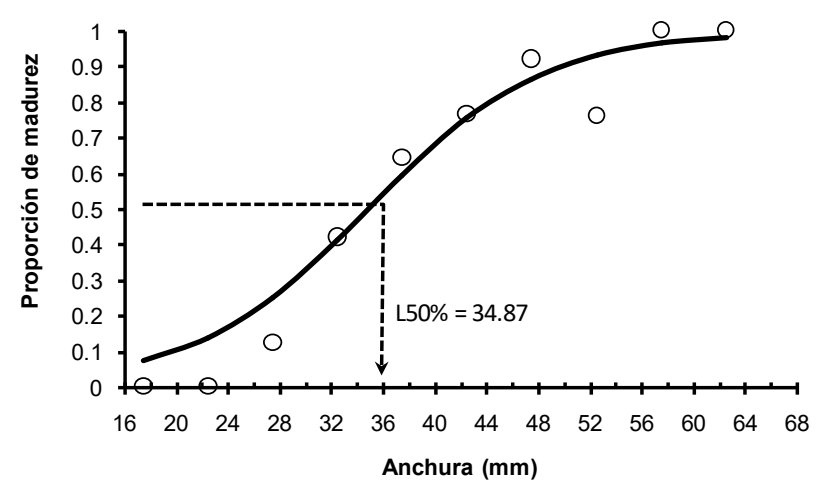

Figura 4. Talla de primera madurez sexual en hembras de G. grapsus en la zona intermareal de las islas del sureste del Golfo de California. 
Tabla 2. Valores máximo, mínimo, promedio y desviación estándar (DE) del (PTH) peso total de hembras, (PSMO) peso seco de la masa ovígera (g) y (FEC) fecundidad (no. de cigotos) de G. grapsus en la zona intermareal de las islas del sureste del Golfo de California.* No se obtuvieron hembras ovígeras.

\begin{tabular}{cccccccccc}
\hline & \multicolumn{3}{c}{ Isla Pájaros } & \multicolumn{3}{c}{ Isla Lobos } & \multicolumn{3}{c}{ Isla Venados } \\
\hline & PTH & PSMO & FEC & PTH & PSMO & FEC & PTH & PSMO & FEC \\
\hline Mar & 46.5 & 0.314 & 20157.9 & 69.96 & 0.239 & 23658.8 & 46.0 & 0.393 & 27778.1 \\
Abr & 60.0 & 0.356 & 23105.8 & 34.02 & 0.260 & 13142.7 & 54.9 & 0.195 & 12785.7 \\
May & 32.1 & 0.288 & 21347.2 & 38.43 & 0.315 & 20475.6 & 43.7 & 0.704 & 42827.5 \\
Jun & 46.2 & 0.515 & 41631.6 & 51.2 & 0.576 & 36335.4 & 42.8 & 0.331 & 16681.1 \\
Jul & 30.5 & $*$ & 12682.4 & 30.5 & 0.324 & 26909.5 & 27.2 & 0.214 & 12682.4 \\
Ago & 31.5 & 0.223 & 14309.3 & 28.6 & 0.310 & 27654.6 & 32.3 & 0.295 & 20188.6 \\
Sep & 32.4 & 0.192 & 15936.1 & 26.7 & 0.278 & 28399.7 & 37.4 & 0.376 & 27694.7 \\
Oct & 25.0 & 0.251 & 21196.1 & 38.0 & 0.249 & 21542.5 & 20.0 & 0.209 & 24381.1 \\
Nov & 36.0 & 0.393 & 38576.7 & 30.1 & 0.340 & 26119.6 & 15.2 & 0.249 & 25808.6 \\
Dic & 43.8 & 0.401 & 37008.9 & $*$ & $*$ & $*$ & $*$ & $*$ & $*$ \\
Ene & 51.5 & 0.410 & 35441.2 & $*$ & $*$ & $*$ & $*$ & $*$ & $*$ \\
Feb & 45.3 & 0.276 & 14733.4 & $*$ & $*$ & $*$ & $*$ & $*$ & $*$ \\
Max. & 60.0 & 0.515 & 38576.7 & 69.9 & 0.576 & 36335.4 & 54.9 & 0.704 & 42827.5 \\
Min. & 25.0 & 0.223 & 12682.4 & 26.7 & 0.239 & 13142.7 & 15.2 & 0.195 & 12682.4 \\
Prom. & 40.1 & 0.329 & 24677.2 & 38.6 & 0.321 & 24915.4 & 35.5 & 0.329 & 23425.3 \\
DE \pm & 10.4 & 0.095 & 10537.1 & 13.9 & 0.101 & 6390.9 & 13.0 & 0.158 & 9386.1 \\
\hline
\end{tabular}

\section{DISCUSIÓN}

Los porcentajes entre sexos de Grapsus grapsus, no presentaron un patrón que permita definir una separación entre la población de hembras y machos, como se ha descrito en otros crustáceos decápodos de interés comercial como Callinectes bellicossus (10), Panulirus inflatus y Panulirus gracilis (14) en algunas de las principales bahías de Sinaloa.

La variación mensual por sexo, corresponde al mayor porcentaje a hembras, para luego su porcentaje declinar, de igual forma se observó el comportamiento en incrementos y decrementos en los porcentajes de captura en los machos. La fluctuación natural en los porcentajes obtenidos entre hembras y machos, posiblemente indica que ambos sexos no compiten por sus espacios o lugares de asentamiento y refugio (2), en este sentido, la población se caracteriza por habitar sustratos rocosos los cuales son comunes en estas islas (12).

La proporción sexual en la población de $G$. grapsus es un indicador de la estabilidad, aún cuando los machos dominaron ligeramente en las colectas en las islas no hubo diferencia significativa en la proporción de sexos. Este hecho esta en concordancia con el porcentaje anual de hembras ovígeras el cual indica que $G$. grapsus se reproduce activamente en esta zona. Sin embargo, en las tres islas se observaron porcentajes distintos de hembras ovígeras y no ovígeras. La isla Pájaros podría ser considerada como una zona de refugio para una reproducción continua del cangrejo roca, debido a que en esta isla, se recolectaron hembras grávidas todo el año, a diferencia de las islas Lobos y Venados donde no se recolectaron hembras ovígeras entre los meses de diciembre y febrero. Lo anterior, debe ser considerado para una regulación pesquera de G. grapsus, e incluso, se debe continuar analizando la población de cangrejo roca en estas islas para determinar que en los meses de diciembre a febrero no se encuentran hembras ovígeras, lo que podría ser considerado entre este período como la principal fase reproductiva de las hembras de G. grapsus y así establecer periodos temporales de protección del recurso (veda).

En las tres islas, las fases embrionarias más representativas fueron las huevas naranja-rojo. Es posible que los embriones durante esta fase de desarrollo permanezcan por un mayor tiempo en incubación, e incluso, representaron el mayor número de huevos (fecundidad) de las hembras ovígeras de G. grapsus comparada con el resto de las fases embrionarias. 
La segunda fase con el mayor porcentaje de huevos fue café-obscuro, que corresponde a cigotos en la última etapa de maduración y que posiblemente eclosionan en un breve tiempo, debido a que el color obscuro de los huevos está relacionado con el desarrollo del ojo naupliar de la larva (16). Además, durante los meses de marzo a agosto en las islas Lobos y Venados, se observó la mayor presencia de estas hembras y en Pájaros disminuyó su porcentaje de recolecta. Entre septiembre y febrero, se detectaron los porcentajes menores de hembras con fase embrionaria café-oscuro. Incluso, se detectó una variación mensual en los porcentajes de hembras por cada fase embrionaria, de tal forma que de manera simultánea se encontraron hembras con cigotos café-oscuro (por desovar) y hembras con huevos en su primera fase embrionaria.

Este trabajo representa el primer estudio de determinación de la talla de primera madurez del cangrejo roca, en el sureste del Golfo de California por lo que puede considerarse un estudio base para futuras investigaciones poblacionales, así como para la formulación de propuestas de manejo pesquero de la especie, siendo de suma importancia para un análisis pesquero de $G$. grapsus en esta zona. En otras especies de cangrejos, han estimado la talla de primera madurez como SantÁna y Pezzuto (17) quienes analizaron en Checeon notialis. Mientras, Becerra Ribeiro et al (18) determinaron una talla mínima de primera madurez en AN en Sesarma rectum de $22.97 \mathrm{~mm}$, ambos estudios realizados en el noroeste de Brasil. En Callinectes sapidus, Severino-Rodrigues et al (19) estimaron una talla mínima de madurez en $103.3 \mathrm{~mm}$ de AN, además estos autores señalaron que la actividad reproductiva de esta especie se presenta a tallas mayores.

Es importante seguir analizando las tallas de primera madurez de G. grapsus en esta zona, ya que podría estar relacionado con la estrategia reproductiva de la población. El señalar una talla de primera madurez en las capturas, puede prevenir evitar que la población sea llevada a una sobreexplotación.

\section{Conflicto de Intereses}

Los autores del presente estudio declaramos que no existe conflicto de intereses con la publicación de este manuscrito.

\section{Agradecimientos}

AI CONACYT por la beca otorgada al primer autor (CVU 90357), por los recursos otorgados a los proyectos SIN-2006-COI-37437 y PROFAPI, 2007-127. Al personal del Cuerpo Académico Consolidado Manejo de Recursos Pesqueros (UAS-CA-2104) y dos revisores por sus valiosas observaciones.

\section{REFERENCIAS}

1. Hernández C, Álvarez F, Villalobos JL. Crustáceos asociados a un sustrato duro en la zona intermareal de Montepio, Veracruz, México. Rev Mex Biodivers. 2010; 81:S141-S151. http://dx.doi.org/10.22201/ ib.20078706e.2010.0.217

2. Arzola González JF, Flores Campaña LM, Vázquez Cervantes A. Crustáceos decápodos intermareales de las islas de la costa de Sinaloa, México. Universidad y Ciencia. 2010; 26(2):179-193. http://era.ujat.mx/ index.php/rera/article/view/175/133

3. Freire AS, Pihneiro MAA, Karam-Silva $H$, Teschima MM. Biology of Grapsus grapsus (Brachyura: Grapsidae) in the Saint Peter and Saint Paul Archipielago Ecuatorial Atlantic Ocean. Helgol Mar Res. 2010; 65(3):263-275. http://dx.doi.org/10.1007/ s10152-010-0220-5
4. Gutiérrez Rubio $Y$, Pérez González R, Campos E, Arzola González JF. Estructura de tallas y relaciones biométricas del cangrejo roca Grapsus grapsus en las islas Lobos, Venados y Pájaros, Sinaloa, México. Hidrobiológica. 2018; 28(1):3136. http://doi.org/10.24275/uam/izt/dcbs/ hidro/2018v28n1

5. Arzola González JF, Flores Campaña LM. Alternativas para el aprovechamiento de los crustáceos decápodos del Estero El Verde Camacho, Sinaloa, México. Universidad y Ciencia. 2008; 24(1):41-48. http://era.ujat. $\mathrm{mx}$ /index.php/rera/article/view/278/229 
6. Johnson ME, Karabinos MP, Mendia V. Quaternary intertidal deposits intercalated with volcanic rocky on Isla Sombrero Chino and Galápagos islands (Ecuador). J Coast Res. 2010; 26(4):762-768. http://dx.doi. org/10.2112/jcoastres-d-10-00010.1

7. Fernández Sánchez E. Efecto del color del oponente en el comportamiento agresivo del Grapsus grapsus (Crustacea: Decapoda). Anl Univ Etol. 2012; 6(1):1-10. http:// etologia.ulpgc.es//revista2012/Elena $\% 20$ Fernandez\%20Sanchez\%201-10.pdf

8. Clores MA, Ramos GA. Reproductive characteristics of the brachyuran crab Grapsus tenuicrustatus (Decapoda: Grapsidae) found in Talim bay, Batangas, Phillippines. Arthopods. 2013; 2(3):111125. http://www.iaees.org/publications/ journals/arthropods/articles/2013-2(3)/ reproductive-characteristics-of-brachyurancrab-grapsus-tenuicrustatus.pdf

9. Pérez-González R. Catch composition of the lobster Panulirus gracilis (Decapoda: Palinuridae) of the western coast Mexico. Lat Am J Aquat Res. 2011; 39(2):225-235. http://dx.doi.org/10.3856/vol39-issue2fulltext-4

10. Rodríguez-Domínguez G, CastilloVargasmachuca S, Pérez-González R, Aragón-Noriega A. Estimation of the individual growth parameters on the brown crab Callinectes bellicossus (Brachyura: Portunidae) using a multi-model approach. Crustaceana. 2012; 85(1):55-69. http:// dx.doi.org/10.1163/156854012X623700

11. Ortega-Lizárraga $G$, Rodríguez-Domínguez G, Pérez-González R, Aragón-Noriega EA. Crecimiento individual y longitud de primera madurez de Callinectes arcuatus en Marismas Nacionales, Nayarit, México. Ciencia Pesquera. 2016; 27(2):3-11. https:// www.gob. $\mathrm{mx} / \mathrm{cms} /$ uploads/attachment/ file/206630/01.pdf

12. Flores Campaña LM, Arzola-González $J F$, Ramírez-Soto $M$, Osorio-Pérez $A$. Repercusiones del cambio climático global en el estado de Sinaloa, México. Cuad Geogr Rev Colomb Geogr. 2012; 21(1):115129. http://dx.doi.org/10.15446/rcdg. v21n1.25562
13. Zar JH. Bioestatistical analysis. Fifth edition. Prentice-Hall Inc: USA; 2010.

14. Arzola-González JF, Flores-Campaña LM, Ortiz-Arellano MA, Gutiérrez- Rubio Y. Captura y aspectos reproductivos de la pesquería de las langostas Panulirus inflatus y $P$. gracilis (Crustacea: Decapoda) en el sur de Sinaloa, México. Revista Ciencia y Mar. 2007; 11(31):15-22. http://servcym. umar.mx/Revista/index.php/cienciaymar/ issue/view/35/ART31 2

15. Escamilla-Montes $R$, De la Cruz-Agüero G, Villalejo-Fuerte MT, Diarte-Plata G. Fecundidad de Callinectes arcuatus y C. bellicosus (Decapoda: Brachyura: Portunidae) en la Ensenada La Paz, Golfo de California, México. Revista Universidad y Ciencia. 2013; 29(1):53-61. http://era. ujat.mx/index.php/rera/article/view/42/42

16. Álvarez $F$, Villalobos $\mathrm{JL}$, Hendrickx ME, Escobar-Briones E, Rodríguez-Almaraz G, Campos E. Biodiversidad de crustáceos decápodos (Crustacea: Decapoda) en México. Rev Mex Biodivers. 2014; 85:S208-S219. http://doi.dx.org/10.7550/ rmb. 38758

17. Sant 'Ana R, Pezzuto PR. Sexual maturity of the deep-sea red crab Chaceon notialis (Brachyura: Gereonydae) in southern Brazil. Lat Am J Aquat Res. 2009; 37(3):429-442. http://doi.dx.org/10.3856/vol37-issue3fulltext-12

18. Becerra Ribeiro F, Matthews Cascon $\mathrm{H}$, Arruda Becerra LE. Morphometric sexual maturity and allometric growth of the grab Sesarma rectum (Crustacea: Sesarmidae) in an impacted tropical mangrove in northeast Brazil. Lat Am J Aquat Res. 2013; 41(2):361-368. http://doi.dx.org/10.3856/ vol41-issue 2 -fulltext-15

19. Severino-Rodrigues $E$, Musiello-Fernandes J, Moura AA, Branco GM, Canéo VO. Fecundity, reproductive seasonality and maturation size of Callinectes sapidus females (Brachyura: Portunidae) in the southeast coast of Brazil. Rev Biol Trop. 2013; 61(2):595-602. https://doi. org/10.15517/rbt.v61i2.11162 